\title{
Análisis de tareas de un libro de texto de primaria desde la perspectiva de los niveles de algebrización
}

\section{Task Analysis of an Elementary School Textbook from the Algebraization Levels Perspective}

\author{
Lilia Patricia Aké Tec ${ }^{1}$ \\ Juan Díaz Godino²
}

\begin{abstract}
Resumen. La introducción del pensamiento algebraico en la educación primaria es una tendencia actual en términos internacionales. Este hecho hace necesario investigar sobre la naturaleza de las tareas planteadas en los libros de texto oficiales de primaria, dado que son un referente para el desarrollo de las clases en dicho nivel educativo. Es en este sentido que el presente trabajo muestra si las tareas del libro de texto de primero de primaria están orientadas a promover el desarrollo del pensamiento algebraico en niños de 6 a 7 años. El estudio se lleva a cabo utilizando el Modelo de Niveles de Algebrización como herramienta de análisis para determinar el carácter algebraico de las tareas, a partir de la práctica matemática que conlleva su resolución. Se reporta que las tareas presentadas en el libro de texto no están intencionalmente dirigidas al desarrollo del pensamiento algebraico en los niños de primero de primaria, pero se evidencia que es posible orientarlas hacia grados progresivos de algebrización.
\end{abstract}

Fecha de recepción: 28 de junio de 2017. Fecha de aceptación: 13 de marzo de 2018.

1 Facultad de Ciencias de la Educación, Universidad de Colima (México). lake86@gmail.com

2 Facultad de Ciencias de la Educación, Universidad de Granada (España). jdgodino@gmail.com; jgodino@ugr.es 
Palabras clave: libros de texto; educación primaria; razonamiento algebraico; niveles de algebrización.

\begin{abstract}
The introduction of algebraic thinking on the elementary school is an actual trend in international terms. This approach arise the need to research the tasks' nature in the official elementary textbooks, because they are fundamental in the course of the lessons at this educational level. This study shows if the official book tasks of the first degree on elementary school are orientated to promote the development of algebraic thinking in children from 6 to 7 years old. The analysis is carried out using the Model of Algebraization Levels as a tool to determine the algebraic character of mathematical tasks, based on the mathematical practice involved in their resolution. The results indicate that the tasks on the elementary official book are not orientated to develop mathematical thinking in children of first degree of elementary school, but it may be orientated towards progressive algebraization degrees.
\end{abstract}

Keywords: Textbook; elementary school; algebraic reasoning; algebraization levels.

\title{
1. INTRODUCCIÓN
}

El álgebra se ha enseñado, tradicionalmente, solo después de que los estudiantes han tenido la oportunidad de adquirir un conocimiento sustancial de la aritmética en la educación primaria. Es decir, el pensamiento aritmético ha sido considerado un requisito previo para la aparición y desarrollo del pensamiento algebraico (Radford, 2012). Por tanto, una introducción del álgebra en la escuela primaria parece contradecir tal suposición. Sin embargo, durante las últimas décadas diversas investigaciones en Educación Matemática apoyan la introducción temprana del álgebra y recomiendan que las actividades algebraicas comiencen en los primeros años y que la notación algebraica empiece a ser introducida entre los grados tercero y quinto de la escuela primaria (Schliemann, Carraher \& Brizuela, 2007).

El difícil acceso conceptual que tiene la mayoría de los estudiantes con esta asignatura es lo que sustenta su introducción durante los primeros años escolares, ya que podría enriquecer e incrementar la compresión de los niños sobre conceptos algebraicos, y así eliminar la tardía y abrupta introducción del álgebra 
de secundaria (Carpenter, Frankie \& Levi, 2003). Esta incorporación del álgebra en la educación primaria no es planteada como asignatura, sino como una manera de desarrollar mayor grado de generalidad en el pensamiento de los alumnos, y mayor capacidad de comunicar dicha generalidad (Lins \& Kaput, 2004). Esta introducción no significa que ahora se enseñe el álgebra de secundaria en la escuela primaria, sino utilizar los contextos que se entrelazan con los temas del currículo de la escuela elemental y poco a poco introducir la notación formal (Carraher, Schliemann \& Schwartz, 2008). Esta propuesta curricular es denominada "Early algebra" por diversos autores. Por otro lado, Kaput en 1998 refiere a esta concepción y denomina "algebrización del currículo" a la tendencia de promover una "nueva álgebra con entendimiento", desarrollando el pensamiento algebraico no solo en la educación primaria, sino en todos los niveles escolares hasta el bachillerato. Algunos avances de esta corriente pueden advertirse en el creciente número de investigaciones que han comenzado a indagar sobre el pensamiento algebraico en niños de la escuela primaria, centrándose principalmente en el estudio de patrones y funciones (Blanton \& Kaput, 2011; Rivera, 2013) y el pensamiento relacional (Carpenter, Franke \& Levi, 2003; Whitacre, Schoen, Champagne \& Goddard, 2017). Los estudios en la escuela secundaria giran en torno a la compresión de las funciones (Ellis, 2011), de la variable y la equivalencia (Alibali, Knuth, Hattikudur, McNeil, \& Sthephens, 2007), por mencionar algunos ejemplos. Sin embargo, aún son pocas las investigaciones que se han centrado en este nivel educativo, y sobre todo en el nivel medio superior.

En México esta tendencia de algebrizar el currículo se ha manifestado en las nuevas reformas que impactan el currículo de la escuela obligatoria, tanto de primaria como de secundaria. Por ello, teniendo como referente que el desarrollo del pensamiento algebraico es una tendencia actual, tanto en las investigaciones (Cai \& Knuth, 2011; Kieran, Pang, Schifter \& Fong, 2016) como en las propuestas curriculares (NCTM, 2000; CCSSM, 2011) se hace necesario indagar sobre la manera en la que se está llevando dicha introducción en México.

El estudio que aquí reportamos es parte de una investigación más amplia para indagar sobre la naturaleza algebraica de las tareas incluidas en los libros de texto oficiales de matemáticas de la educación obligatoria en México, utilizados de manera homogénea en todo el país. Este estudio cobra relevancia porque la enseñanza de las matemáticas se basa en los libros de texto más que en cualquier otra área de estudio, además de que también dominan las percepciones y prácticas de las matemáticas escolares (Jamieson-Proctor \& Byrne, 2008), al menos en México. En concreto, se discute si las tareas de un libro de texto de 
primaria presentan rasgos que promuevan el pensamiento algebraico. Para ello se implementa el modelo de Niveles de Algebrización (Aké, Godino, Gonzato \& Wilhelmi, 2013; Godino, Aké, Gonzato \& Wilhelmi, 2014) articulado desde el marco del Enfoque Onto-Semiótico de la Cognición e Instrucción Matemáticos (Godino, Batanero \& Font, 2007), en el cual se proponen criterios que permiten discernir entre actividades matemáticas no algebraicas, proto-algebraicas y algebraicas. Se pretende evidenciar que el modelo de niveles de algebrización puede usarse como herramienta de análisis, tanto para determinar el nivel de algebrización de la actividad matemática de los estudiantes como para analizar las tareas presentes en los libros de texto de la educación básica en México.

A continuación, en el segundo apartado presentamos el modelo de algebrización en el cual se sustentan los análisis elaborados sobre el libro de texto. El tercer apartado describe el contexto educativo mexicano y la organización de los libros de textos oficiales utilizados en las aulas. La cuarta parte muestra el análisis realizado a un libro de texto de educación primaria, el de primer grado. Finalmente, en el apartado cinco presentamos algunas reflexiones e implicaciones para la formación de maestros de educación primaria.

\section{NIVELES DE ALGEBRIZACIÓN}

La introducción del pensamiento algebraico en la escuela primaria es una propuesta que pretende hacer explícito el carácter algebraico de los temas existentes en la matemática escolar. Esto es posible, tal y como lo señalan Carraher \& Schliemann (2007), debido a que el álgebra está implícita dentro del currículo de la matemática de la escuela primaria. Al respecto, varios expertos en didáctica del álgebra han investigado el desarrollo del pensamiento algebraico atendiendo diferentes formas que advierten como rutas de acceso (Butto \& Rojano, 2004), tales como el pensamiento relacional, el pensamiento proporcional, los patrones y el pensamiento funcional, entre otras. Sin embargo, la mayoría de dichas investigaciones son disjuntas y sin ningún consenso sobre cuáles son los rasgos característicos del álgebra que debieran considerarse para el desarrollo del pensamiento algebraico a lo largo del currículo escolar (Carraher \& Schliemann, 2007).

Como respuesta, en el marco del Enfoque Onto-Semiótico se ha articulado un modelo basado en el reconocimiento de distintos niveles de algebrización. El modelo implica una visión ampliada de la naturaleza del álgebra escolar, y propone una diferenciación de cuatro niveles para la actividad algebraica. El 
nivel cero indica ausencia de rasgos algebraicos, mientras que los siguientes dos niveles de algebrización primarios sugieren niveles incipientes o proto-algebraicos. Finalmente, un tercer nivel indica que la actividad matemática puede considerarse propiamente algebraica. A continuación, se describen estos niveles de algebrización, recogidos de los trabajos de los autores.

1. Nivel cero de algebrización: intervienen objetos extensivos (particulares) expresados mediante lenguajes natural, numérico, icónico o gestual. Pueden intervenir símbolos que refieren a un valor desconocido, pero dicho valor se obtiene como resultado de operaciones sobre objetos particulares. En tareas estructurales no se reconocen propiedades, mientras que en las tareas funcionales simplemente se determina una regla recursiva.

2. Nivel uno de algebrización: intervienen objetos intensivos (generales) cuya generalidad se reconoce de manera explícita mediante lenguajes natural, numérico, icónico o gestual. Pueden intervenir símbolos que refieren a los intensivos reconocidos, pero sin operar con dichos objetos. Se identifican propiedades, equivalencias numéricas y relaciones a partir de tareas estructurales, mientras que en tareas de funciones se expresa una regla general.

3. Nivel dos de algebrización: intervienen cantidades indeterminadas o variables expresadas con lenguaje simbólico o simbólico-literal para referir a los intensivos reconocidos, aunque ligados a la información del contexto espacial-temporal. Se realizan tratamientos con las incógnitas para resolver ecuaciones del tipo $A x \pm B=C$ y la formulación de una regla general en el contexto de las funciones.

4. Nivel tres de algebrización: son generados objetos intensivos representados de manera simbólica-literal, y se opera con ellos; se elaboran transformaciones en la forma simbólica de las expresiones, conservando la equivalencia. Se realizan tratamientos con las incógnitas para resolver ecuaciones del tipo $A x \pm B=C x \pm D$, y la formulación simbólica y descontextualizada de reglas canónicas de expresión de funciones.

Desde el Enfoque Onto-Semiótico se sugiere que los objetos algebraicos inmersos en el pensamiento funcional y estructural, que pueden estar o no en un contexto de modelización, van adquiriendo un significado diferente conforme haya una "evolución" en los procesos de generalización, simbolización y transformación o cálculo. Por ejemplo, el conocimiento de propiedades estructurales, en un nivel elemental, comprende el conocimiento de propiedades como la asociativa, 
conmutativa y otras (Warren, 2001). En grados intermedios, se reconoce con el tratamiento de la equivalencia a través de "ecuaciones numéricas" enmarcadas dentro del pensamiento relacional (Carpenter et al., 2003). Posteriormente, en grados más avanzados forman parte de un conocimiento de la estructura y la equivalencia de las ecuaciones subsecuentes que se obtiene al resolver una ecuación reconociendo "formas" matemáticas existentes entre los elementos, lo que los autores denominan sentido estructural (Hoch y Dreyfus, 2004). Para el caso de las funciones, en los niveles elementales puede asociarse con el reconocimiento de patrones y relaciones de cambio entre dos conjuntos que conducen a la generación de una regla general, la cual puede ser formulada en un lenguaje verbal, numérico e incluso gestual (Radford, 2003). En niveles posteriores se asocia este reconocimiento a la formulación de la regla general en un lenguaje alfanumérico, mientras que en etapas más avanzadas el alumno tendría que ser capaz de operar y transformar las expresiones en otras equivalentes.

\section{EL RAZONAMIENTO ALGEBRAICO EN EL CONTEXTO EDUCATIVO MEXICANO}

La educación básica en México está regida por la Secretaria de Educación Pública (SEP) y se ha ido modificando desde el plan de estudios de 1958 hasta la Reforma Integral del Educación Básica (RIEB) del año 2009, la cual continúa vigente. Dicha reforma actual integra los niveles preescolar, primaria y secundaria en cuatro periodos escolares (Tabla 1), y organiza los contenidos de la educación básica en Estándares Curriculares que se desarrollan en cada uno de esos periodos. De esta manera, se cuenta con 6 estándares curriculares: estándares de español, habilidad lectora, segunda lengua inglés, matemáticas, ciencias y, finalmente, estándares de habilidades digitales.

Tabla 1. Periodos escolares en los que se divide la educación básica (SEP, 2011: 42).

\begin{tabular}{ll}
\hline Periodo escolar & Edad aproximada \\
\hline Primero: Primer a tercer grado de preescolar & Entre 5 y 6 años \\
Segundo: Primer a tercer grado de primaria & Entre 7 y 9 años \\
Tercero: Cuarto a sexto grado de primaria & Entre 10 y 12 años \\
Cuarto: Primer a tercer grado de secundaria & Entre 13 y 15 años \\
\hline
\end{tabular}


Los estándares curriculares de matemáticas "representan la visión de una población que sabe utilizar los conocimientos matemáticos" (SEP, 2011: 88); están organizados en 4 ejes temáticos: (1) Sentido numérico y pensamiento algebraico; (2) Forma, espacio y medida; (3) Manejo de la información; (4) Actitud hacia el estudio de las matemáticas.

Llama la atención la inclusión del "pensamiento algebraico" en el primer eje temático, que se corresponde con las nuevas tendencias sobre introducir formas de razonamiento algebraico en la educación básica en México y, por consiguiente, en la escuela primaria. En este eje se alude al pensamiento algebraico como parte del trayecto formativo, desde preescolar hasta la educación secundaria, en el cual destacan los fines más relevantes del estudio de la aritmética y el álgebra, tales como la modelización de situaciones mediante el uso del lenguaje aritmético, la exploración de propiedades aritméticas que en la secundaria podrán generalizarse con el álgebra y la puesta en juego de diferentes formas de representar y efectuar cálculos (SEP, 2011). Así, nuestro interés de profundizar en el enfoque sobre el pensamiento algebraico en la escuela primaria mexicana, a través del estudio de las tareas del libro de texto, obedece a que las consideraciones sobre este eje refieren a la exploración de propiedades y procesos de generalización y modelización, aspectos que, según las investigaciones, constituyen algunos de los rasgos característicos de la actividad algebraica (Kaput, 1998; Kieran, Pang, Schifter \& Fong, 2016).

Con esta introducción es necesario esclarecer, entre otras cuestiones, la naturaleza más o menos algebraica de las tareas incluidas en los libros de texto de matemáticas de la educación primaria, es decir, resulta preciso establecer cuándo una tarea matemática puede promover un pensamiento algebraico. Este hecho quizá resulte claro y definido cuando nos referimos al álgebra de secundaria, entendida de una manera restrictiva como lenguaje alfanumérico, y orientada básicamente a la resolución de ecuaciones y estudio de los polinomios. Sin embargo, cuando hacemos referencia a las tareas planteadas en la escuela primaria, el determinar su naturaleza algebraica parece difuso ya que, tradicionalmente, el foco de las matemáticas de primaria ha sido profundamente orientado hacia la aritmética y el cálculo, con pequeña atención a las relaciones y estructura (Kaput \& Blanton, 2002), que son la base para el desarrollo de un pensamiento algebraico en los niños. De esta manera, se requiere evaluar los libros de texto de matemáticas de la educación primaria, pues son considerados una fuente de contenido y actividades de instrucción para los profesores (Jamieson-Proctor \& Byrne, 2008). Por ello, es preciso 
determinar si las tareas priorizan los aspectos procedimentales en vez de fomentar los aspectos estructurales y funcionales necesarios para la comprensión del álgebra, determinando así si son potencialmente útiles para el desarrollo del pensamiento algebraico.

En el caso de México, los libros de texto para la educación primaria están regidos por la Secretaría de Educación Pública (SEP). En el aula se trabajan 2 tipos: el de desafíos matemáticos, compendio de tareas propuestas para el alumno en la cotidianeidad de la clase, y el libro guía para el profesor, que integra los contenidos a desarrollar durante el curso. El de desafíos matemáticos está organizado en bloques, y cada bloque tiene un número determinado de tareas. Cada tarea presenta consignas que indican al estudiante las instrucciones para desarrollarla. Por otro lado, el libro guía para el profesor también se organiza en bloques, y cada bloque está estructurado en cuatro secciones. La primera sección es de "lección", en la que se desarrolla el contenido matemático a través de actividades grupales e individuales. En la segunda sección, “¿qué aprendí?", el profesor motiva al alumno a resolver los problemas relacionados con los aprendizajes del bloque. Las secciones tercera y cuarta se corresponden con las propuestas para la "evaluación" y la "autoevaluación", respectivamente.

Respecto a los libros de texto, los maestros necesitan estar capacitados en utilizarlos como medios para el desarrollo del pensamiento algebraico en los niños y promover -a través de las tareas que ahí se presentan- conjeturas, generalizaciones y justificaciones de hechos y relaciones matemáticas. Además, deben ser capaces de crear un ambiente escolar en el cual se valore que los alumnos exploren, modelicen, discutan, argumenten y comprueben ideas (Blanton \& Kaput, 2011).

\section{ANÁLISIS DE UN LIBRO DE TEXTO DE LA EDUCACIÓN PRIMARIA}

Como mencionamos, el trabajo que aquí se desarrolla es parte de un estudio más amplio sobre el análisis de libros de texto oficiales de la educación obligatoria en México. Para efectuar el estudio elegimos los libros de desafíos matemáticos porque son los materiales de trabajo de los niños. Lo que aquí reportamos es el análisis del libro de primer grado de educación primaria, organizado en cinco bloques, con un total de 57 tareas (SEP, 2015). 


\subsection{MÉTODO}

El presente trabajo de tipo cualitativo (Vasilachis, 2009) se interesa en analizar las tareas del libro de texto de primero de primaria atendiendo a 4 criterios del modelo de Niveles de Algebrización (Godino et al., 2014): (1) Tipo de objetos algebraicos; (2) Tipo de notación o representaciones; (3) Transformaciones o cálculo analítico; y (4) Situaciones de modelización.

Para llevar a cabo el análisis elaboramos una primera categorización a través de la identificación de objetos algebraicos de carácter estructural (propiedades de las operaciones, equivalencias, cantidades desconocidas o indeterminadas que pueden representar incógnitas o variables, etc.), y de carácter funcional (relaciones de cambio, patrones, sucesiones). Identificamos también el tipo de notación o representaciones que se manifiestan en la tarea, o sea, de tipo verbal, numérico, tabular, icónico, etc., que son necesarias para su resolución. Aludimos también al tratamiento que se aplica a las cantidades desconocidas, en el sentido al que refieren Puig \& Rojano (2004), de operar con lo indeterminado. El tema de la modelización fue considerado en términos de Filloy, Puig \& Rojano (2008), quienes sugieren que los problemas verbales se modelizan a través de ecuaciones. Es importante mencionar que el modelo de algebrización propuesto por Godino et al, (2014) establece que el carácter algebraico se refiere a la actividad matemática del estudiante y no a la tarea en sí, es decir, se basa en la actividad efectivamente desarrollada por los sujetos, por lo que su carácter es funcional y contextual (Godino, Castro, Aké \& Wilhelmi, 2012; Godino, et al., 2015). Sin embargo, también es de utilidad para discernir entre tareas que pueden favorecer el pensamiento algebraico, tal y como se ejemplifica en los siguientes análisis cualitativos de las tareas matemáticas que propone el libro de primero de primaria.

\subsection{ANÁlisis DE LAS tAREAS DEL BLOQUE 1 DEL LIBRO DEL ALUMNO}

En el primer bloque del libro para el alumno, que cuenta con 16 tareas, encontramos los siguientes objetos algebraicos (OA): la noción de equivalencia (EQ), cantidad desconocida (CD), patrones (PA) y sucesión (SU) como puede apreciarse en la Tabla 2. 
Tabla 2. Objetos algebraicos identificados en el primer bloque del libro del alumno.

\begin{tabular}{lclc}
\hline \multicolumn{1}{c}{ Tareas } & OA & \multicolumn{1}{c}{ Tareas } & OA \\
\hline Tarea 1. ¿Son iguales? & - & Tarea 9. Competencias & SU \\
Tarea 2. ¿Más o menos? & - & Tarea 10. Formas y colores & PA \\
Tarea 3. ¿Cuántos faltan? & - & Tarea 11. Juego con figuras & - \\
Tarea 4. iVamos a contar! & - & Tarea 12. Quitar y poner & EQ \\
Tarea 5. iContar para atrás! & - & Tarea 13. ¿Cómo quedó? & CD \\
Tarea 6. El calendario & - & Tarea 14. Lo que falta & EQ \\
Tarea 7. iLeo y escribo números! & CD; SU & Tarea 15. iA rodar la pelota! & - \\
Tarea 8. Contemos frijolitos & - & Tarea 16. ¿Qué hago dentro y fuera de la & - \\
& & escuela? & \\
\hline
\end{tabular}

Además, predominan las representaciones de tipo numérica, icónica y tabular en las tareas; naturalmente, no se les da un tratamiento analítico a las cantidades desconocidas, pues no se opera en ambos lados de las expresiones numéricas que son planteadas. La modelización implicada en este primer bloque refiere a problemas verbales de suma y resta en las que el elemento desconocido es el resultado. Las tareas no tienen la intención inmediata de situar a los estudiantes en contextos de análisis de propiedades y relaciones entre cantidades o conjuntos, pero es posible orientarlas para favorecer la emergencia del pensamiento algebraico en los niños. En este bloque la tarea 10 resultó promotora de un pensamiento proto-algebraico de carácter funcional, mientras que las tareas 12 y 14 de carácter estructural. Por ejemplo, la tarea 10 (Figura 1) está enfocada en el conteo de las piezas que conforman la figura y a reproducirlas de acuerdo al modelo dado, sin dirigir al estudiante hacia la apreciación de relaciones entre las características de la secuencia de figuras. Sin embargo, es posible identificar en la tarea la idea de patrón que puede comenzar a desarrollarse, en un primer momento, al asociar una posición para cada figura y pensar en el término siguiente. Aunque la acción anterior implica un nivel cero de algebrización, a partir de esta situación puede orientarse hacia un primer nivel de pensamiento proto-algebraico, preguntándole a los alumnos sobre las características de las figuras en las posiciones pares e impares, y que finalmente generalicen a la posición n-ésima. 


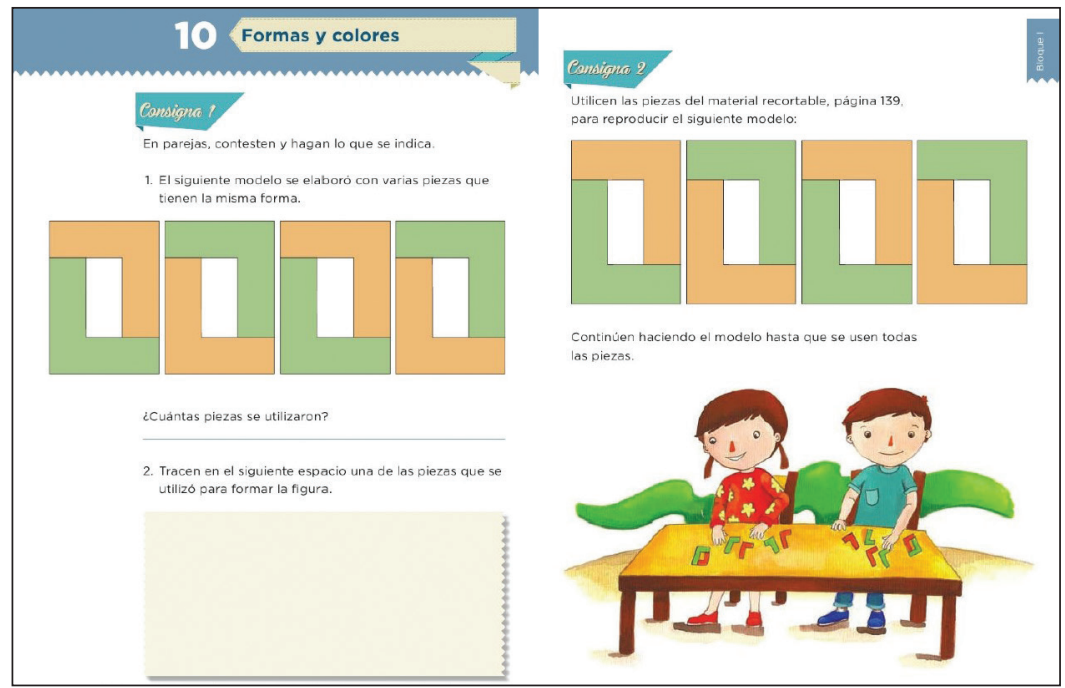

Figura 1. Ejemplo de tarea en el libro de texto de primaria (SEP, 2015: 26).

El alumno tiene que asociar la posición $(N=1,2,3,4, \ldots)$ para cada figura con el color de los elementos que la conforman (Figura 2). Si identifica, por ejemplo, que a todos los números pares le corresponde siempre la figura cuyo color verde está "arriba" y a todos los números impares le corresponde siempre la figura cuyo color verde está "abajo", entonces se puede decir que la tarea promueve un pensamiento proto-algebraico de carácter funcional de nivel 1 de algebrización. Claramente el niño de 6-7 años no tiene que enunciar la regla verbalmente, basta con que sea capaz de formar la figura correctamente si se le solicita la posición 15 o 26, sin que tenga que formar las figuras precedentes.

\begin{tabular}{|c|c|c|c|c|c|c|}
\hline Figura & & & & & & $\cdots$ \\
\hline Posición & 1 & 2 & 3 & 4 & 5 & 7 \\
\hline
\end{tabular}

Figura 2. Tratamiento proto-algebraico funcional de la tarea planteada en la Figura 2.

Por otro lado, la tarea 12 (Figura 3) propone al alumno el conteo de los objetos. En el primer caso de la consigna 1, el niño tiene que contar los jarrones de la 
mesa naranja para determinar el número de objetos que están sobre ella, es decir 9; luego tendría que iniciar el conteo de los jarrones de la mesa verde, o sea 5, y continuar hasta que llegue a 9. Así obtendría la respuesta. De manera similar puede resolver lo que se indica en la consigna 2, coloreando las frutas de la canasta amarilla. Realizar este conteo no implica un acercamiento a formas de pensamiento proto-algebraicas, pero de la tarea emerge la noción de equivalencia, que es posible potenciar para promover el pensamiento algebraico, proporcionando otras indicaciones al estudiante.

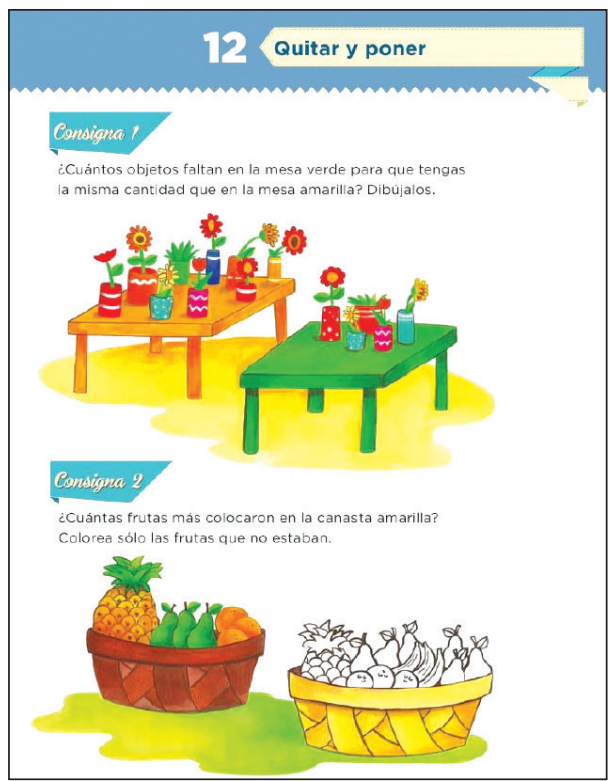

Figura 3. Ejemplo de tarea en el libro de texto de primaria (SEP, 2015: 29).

A partir de la tarea 12 es posible establecer una correspondencia entre las flores de la mesa verde y las de la mesa naranja, sin hacer el conteo de manera explícita. Posteriormente, recurrir a la descomposición numérica del número de objetos que hay sobre la mesa naranja y conducir hacia un pensamiento relacional del signo igual (Figura 4). 


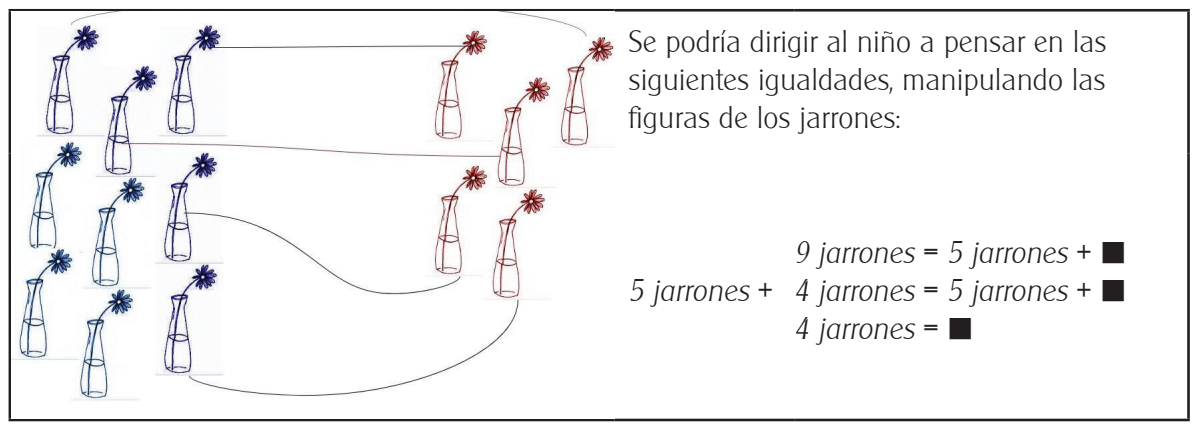

Figura 4. Tratamiento proto-algebraico estructural de la tarea planteada en la Figura 3.

De esta manera se usa el sentido del número y la operación para reflexionar sobre expresiones matemáticas en calidad de objetos, en lugar de como procedimientos aritméticos que se llevan a cabo (Carpenter, Franke \& Levi, 2003). Lo anterior situaría la tarea como promotora de un pensamiento proto-algebraico de carácter estructural de nivel 1 de algebrización.

En las tareas 7, 9 y 13, particularmente, aunque se identificaron objetos algebraicos, estos no resultaron ser condición suficiente para desarrollo del pensamiento algebraico. En la tarea 7 y 9 se identificó la noción de sucesión, pero la tarea refiere al conteo de los primeros 30 números naturales completando casillas vacías (cantidad desconocida) consecutivas, por lo cual estos dos elementos -sucesión y cantidad desconocida- presentes en las tareas, no potencian el pensamiento proto-algebraico. Para el caso de la tarea 13 observamos que la sola existencia de cantidades indeterminadas tampoco promueve un pensamiento algebraico, necesariamente tiene que estar en un contexto de equivalencia. A continuación, se ejemplifica esta situación con la Figura 5 que representa la tarea 13, la única del bloque 1 que plantea problemas de tipo verbal. 


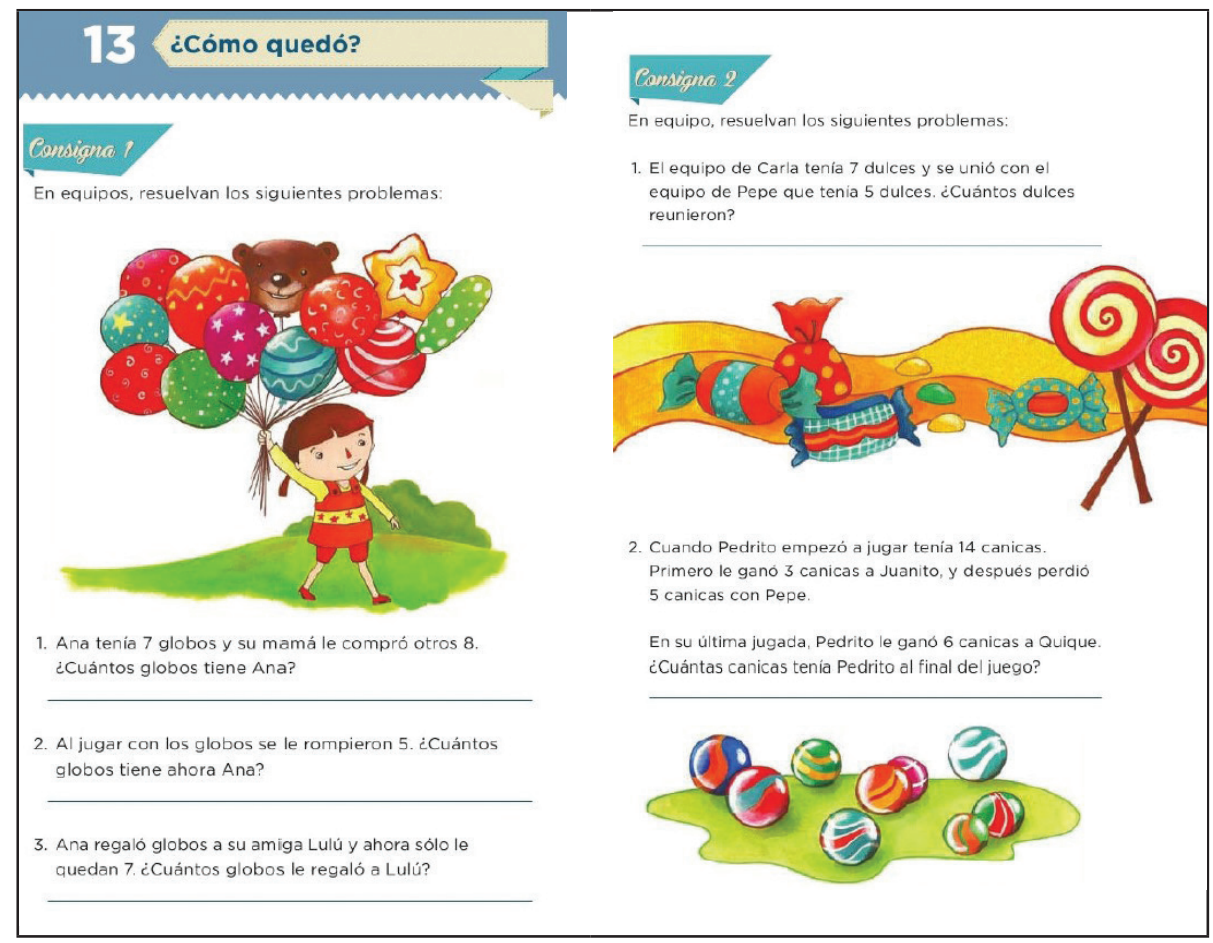

Figura 5. Ejemplo de tarea en el libro de texto de primaria (SEP, 2015: 30).

En esta tarea 13 se promueve la idea del signo igual en su significado como resultado de operación, por lo cual la cantidad desconocida queda asociada a un carácter procedimental que denota la expresión $a+b=\mathbf{a}$, con $a$ y $b$ conocidos. La solución implica el uso de operaciones aritméticas encadenadas $e$ indicadas en el enunciado del problema, por lo que no es posible implicar al estudiante en la comprensión y aplicación de propiedades de las operaciones.

\subsection{ANÁLISIS DE LAS TAREAS DEL BLOQUE 2 DEL LIBRO DEL ALUMNO}

En el segundo bloque del libro de primer año, que cuenta con 12 tareas, encontramos objetos algebraicos tales como la noción de equivalencia (EQ), propiedades de las operaciones (PO) y cantidades desconocidas (CD), presentes la Tabla 3. 
Tabla 3. Objetos algebraicos identificados en el segundo bloque del libro del alumno.

\begin{tabular}{lclc}
\hline \multicolumn{1}{c}{ Tareas } & OA & \multicolumn{1}{c}{ Tareas } & OA \\
\hline Tarea 17. Carrera de autos & - & Tarea 23. iCuántos más pinto? & CD \\
Tarea 18. Animales en orden & - & Tarea 24. El camión & CD \\
Tarea 19. ¿Quién juntó más dinero? & EQ; PO & Tarea 25. Quita y pon & - \\
Tarea 20. La juguetería & EQ & Tarea 26. Juanito el dormilón & CD \\
Tarea 21. iA igualar cantidades! & EQ; PO & Tarea 27. ¿Hay alguna mal? & - \\
Tarea 22. iCuánto cambio queda? & CD & Tarea 28. ¿Cuándo usar +, -, =? & CD \\
\hline
\end{tabular}

Predominan las representaciones icónicas y es evidente de nuevo que la presencia de las cantidades indeterminadas no es condición suficiente para el desarrollo del pensamiento algebraico. Llama la atención que este bloque presenta 3 tareas que plantean problemas verbales, las tareas 22, 24 y 28. Sin embargo, estos problemas verbales se resuelven con expresiones del tipo $a+b=\mathbf{\square}$, en donde la cantidad inderminada es el resultado de la operación. Lo anterior puede apreciarse en la Figura 6, que muestra un extracto de la tarea 22 en donde la solución al problema verbal planteado resulta de realizar 20 menos 12.

Juan fue a comprar un kilo de azúcar que cuesta $\$ 12$ y su mamá le dio un billete de $\$ 20$ pesos para pagar. ¿Cuánto debe recibir de cambio?

Figura 6. Extracto de la tarea 22 del libro de texto de primaria (SEP, 2015: 44).

Así, encontramos 3 tareas a través de las cuales se puede tener un acercamiento al pensamiento proto-algebraico de carácter estructural, las tareas 19, 20 y 21. A continuación describimos el análisis de la tarea 19 (Figura 7), la cual es planteada con la finalidad de que el niño compare cantidades haciendo la operación de sumar números de hasta dos dígitos. 


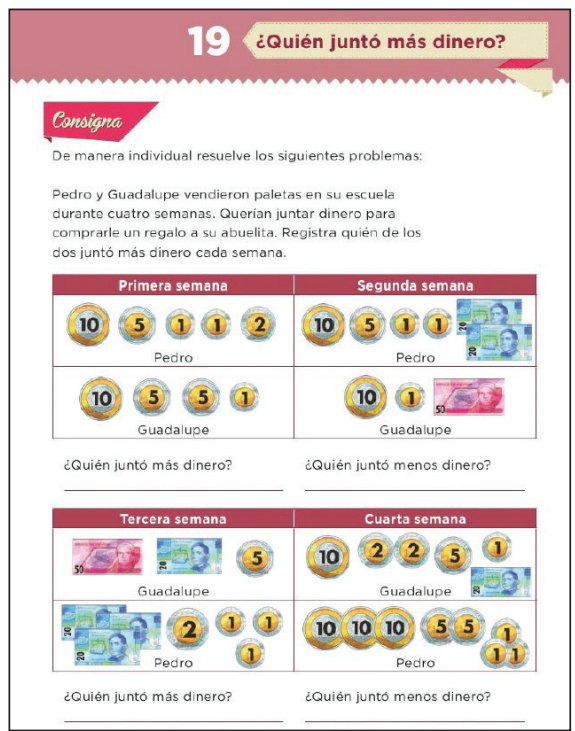

Figura 7. Ejemplo de tarea en el libro de texto de primaria (SEP, 2015: 41).

En la tabla que corresponde a la primera semana, se cuenta el dinero total de Pedro $(10+5+1+1+2=19)$, para compararlo con el dinero total de Guadalupe $(10+5+5+1=21)$. Claramente es promovida una interpretación de signo igual como resultado de operación, además de calcular con números particulares, en un formato icónico y aplicando la correspondiente consigna aditiva para hallar la respuesta. No obstante, con esta misma situación que plantea la tarea podría orientarse la atención del niño en el uso de la propiedad asociativa, a través de la descomposición numérica y de la noción de equivalencia. Por ejemplo, la Figura 8 representa el tratamiento que podría darse al dinero acumulado de la primera semana de Guadalupe y Pedro. Es claro que el niño no tiene que implicarse en el nombramiento de cada una de las propiedades, pero si se le puede referir a la acción que conlleva su uso, lo cual implica un pensamiento proto-algebraico de carácter estructural de nivel 1 de algebrización. Posteriormente, si se amplía al marco del estudio de las equivalencias tales como $5=1+1+1+2$, $1+1+1+2=(1+1+2)+1$ y $4+1=1+1+1+2$, en las que se comience a introducir la notación simbólica, se estaría en un nivel de algebrización 2. Por ejemplo: $4+\boldsymbol{\square}=\boldsymbol{\square}+\boldsymbol{\square}+\boldsymbol{\square}+2$. De la misma manera puede analizarse el dinero obtenido por Pedro, para finalmente comparar sentencias como $10+5+4$ y $10+5+4+2$ y así obtener la respuesta solicitada en la tarea. 


\begin{tabular}{|lll|}
\hline Dinero de Guadalupe & $10+5+5+1$ & Planteamiento inicial \\
& $10+5+1+1+1+2+1$ & Descomposición numérica del número \\
& $10+5+(1+1+2)+(1+1)$ & 5 \\
$10+5+4+2$ & Asociativa \\
& & Suma \\
Dinero de Pedro & $10+5+1+1+2$ & Planteamiento inicial \\
& $10+5+(1+1+2)$ & Asociativa \\
& $10+5+4$ & Suma \\
\hline
\end{tabular}

Figura 8. Tratamiento proto-algebraico estructural de la tarea propuesta en la Figura 7.

De esta forma se incursiona al niño en el conocimiento de las propiedades estructurales que comprende el conocimiento, en este caso propiedades como la asociativa; también en el sentido relacional del signo igual para el tratamiento de las equivalencias a través de identidades numéricas, e introducir la notación simbólica. El uso de identidades aritméticas puede considerarse un paso previo en la adquisición del concepto de ecuación (Alibali et al., 2007).

\subsection{ANÁlisis de LAS tAREAS DEL BLOQUe 3 DEL LIBRO DEL ALUMNO}

En el tercer bloque, que cuenta con 12 tareas, fueron encontrados los siguientes objetos algebraicos: la noción de equivalencia (E), propiedades de las operaciones (PO), cantidades desconocidas (CD) y sucesión (SU), como puede apreciarse en la Tabla 4.

Tabla 4. Objetos algebraicos identificados en el tercer bloque del libro del alumno.

\begin{tabular}{|c|c|c|c|}
\hline Tareas & $\mathrm{OA}$ & Tareas & $\mathrm{OA}$ \\
\hline Tarea 29. Tarjetas ordenadas & - & Tarea 35. Historias con números & $C D$ \\
\hline $\begin{array}{l}\text { Tarea 30. Todos contamos y } \\
\text { contamos todos }\end{array}$ & $\mathrm{CD} ; \mathrm{SU}$ & Tarea 36. Las granjas & $C D$ \\
\hline Tarea 31. Un mensaje para el rey & SU & Tarea 37. Inventa una historia & $\mathrm{CD}$ \\
\hline Tarea 32. Encuentra el número & $\mathrm{CD} ; \mathrm{SU}$ & Tarea 38. Del más corto al más largo & PO \\
\hline Tarea 33. iPiensa pronto! & $\mathrm{CD} ; \mathrm{PO} ; \mathrm{EQ}$ & Tarea 39. Cerca o lejos, ¿de qué? & - \\
\hline Tarea 34. ¿Con cuántas se puede? & $\mathrm{CD} ; \mathrm{PO} ; \mathrm{EQ}$ & & \\
\hline
\end{tabular}


Prevalecen las representaciones numéricas y tabulares; al igual que en el bloque anterior, encontramos 3 tareas, 35, 36 y 37, en contextos de problemas verbales que hacen alusión al tipo de modelización que se proponen a través de ellas. Estas tareas evocan cantidades indeterminadas que se corresponden con los resultados de las operaciones del tipo $a+b=\mathbf{G}$, por lo cual tampoco implican el desarrollo de un pensamiento algebraico. En este bloque hay un aumento entre el número de tareas que evocan cantidades indeterminadas que se corresponden con los resultados de las operaciones, por ello no se promueve la analiticidad con las cantidades indeterminadas. Las tareas 30, 31 y 32 están orientadas al conteo de los primeros 100 números naturales de forma consecutiva; en el caso de las tareas 30 y 31, ocultan valores que el niño tendría que completar. Al igual que la tarea 7 del bloque 1, estas tareas no promueven el pensamiento pro-algebraico.

Identificamos, finalmente, a las tareas 33, 34 y 38 como facilitadoras para el desarrollo del pensamiento proto-algebraico de carácter estructural. Las tareas 33 y 34 se enmarcan en la descomposición numérica con objeto de obtener expresiones equivalentes para un mismo número. Por ejemplo la tarea 34, presentada en la Figura 9, se encuentra articulada con la intención de descomponer los números 15 y 4, y encontrar las expresiones que cumplan con la condición $x+y=15$ y $x-y=4$.

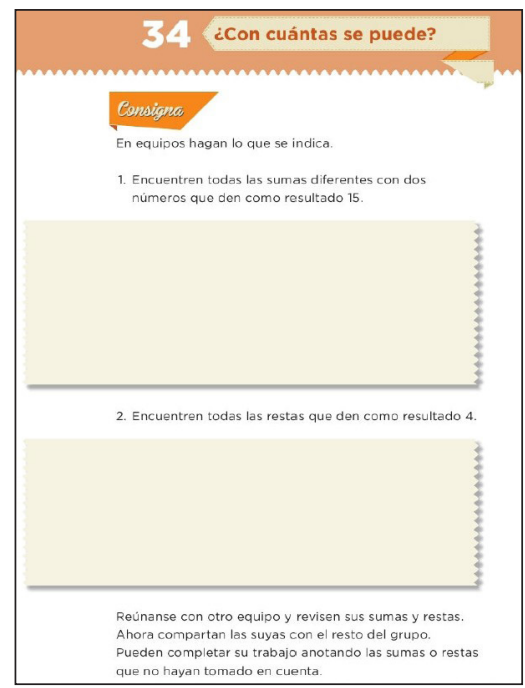

Figura 9. Ejemplo de tarea en el libro de texto de primaria (SEP, 2015: 66). 
La tarea sugiere una interpretación del signo igual como resultado de operación, ya que no se propone al estudiante que compare las expresiones equivalentes entre sí. Sin embargo, es posible dar a la tarea dos orientaciones en el aula. En la primera, el profesor podría generar el análisis de las diferentes sentencias numéricas que expresen la suma de dos dígitos igual a 15 , por ejemplo: $1+14=15 ; 2+13=15 ; 3+12=15$ y así sucesivamente. Posteriormente, podrían analizarse las equivalencias entre dichas expresiones, es decir, $1+14=$ $2+13 ; 1+14=3+12 ; 2+13=3+12$, utilizando la propiedad asociativa. Es en este sentido en el que se plantea un cambio en la enseñanza de contenidos aritméticos en la educación primaria respecto al desarrollo de un significado relacional y simétrico del signo igual, pues los niños de este nivel tienden a ver este símbolo como un operador que indica un resultado (Jones \& Pratt, 2006). Con el análisis de expresiones numéricas equivalentes estaríamos promoviendo pensamiento proto-algebraico de carácter estructural de nivel 1, que podría considerarse de un nivel 2 de algebrización si se introduce notación simbólica (de manera similar a la tarea 19 del bloque 2).

La segunda orientación implicaría un análisis tabular como el dado en la Figura 10, donde la representación tabular podría ampliarse al estudio de los números negativos y de expresiones equivalentes, tales como $y=15-x y$ $y=x-4$. También podría implicarse el estudio de las gráficas correspondientes a las funciones definidas, que enmarcaría a la tarea como promotora de un pensamiento algebraico consolidado con un nivel 3 de algebrización.

De la siguiente tabla, ¿cuáles son los valores que pueden tomar los dos números desconocidos para que su suma sea 15 ?

\begin{tabular}{|c|r|r|r|r|r|r|r|r|r|r|r|r|r|r|r|}
\hline$x$ & 0 & 1 & 2 & 3 & 4 & 5 & $\ldots$ & 8 & 9 & 10 & 11 & 12 & 13 & 14 & 15 \\
\hline$y$ & 15 & 14 & 13 & 12 & 11 & 10 & $\ldots$ & 7 & 6 & 5 & 4 & 3 & 2 & 1 & 0 \\
\hline$x+y$ & 15 & 15 & 15 & 15 & 15 & 15 & $\ldots$ & 15 & 15 & 15 & 15 & 15 & 15 & 15 & 15 \\
\hline
\end{tabular}

Figura 10. Tratamiento proto-algebraico funcional de la tarea propuesta en la Figura 10.

Con esta tarea se puede tratar a las operaciones aritméticas como funciones, para que los estudiantes analicen las relaciones de cambio en conjuntos de números (Carraher, Schliemann \& Brizuela, 2000). 


\subsection{ANÁlisis de LAS tAREAS DEL BLOQUE 4 DEL LIBRO DEL ALUMNO}

El bloque 4 del libro cuenta con 12 tareas y en él se encontraron objetos algebraicos como la noción de equivalencia (EQ), propiedades de las operaciones (PO), cantidades desconocidas(CD), la noción de relación de cambio (RC) y sucesión (SU), mostrados en la tabla siguiente:

Tabla 5. Objetos algebraicos identificados en el cuarto bloque del libro del alumno.

\begin{tabular}{lllc}
\hline \multicolumn{1}{c}{ Tareas } & \multicolumn{1}{c}{$\mathrm{OA}$} & \multicolumn{1}{c}{ Tareas } & OA \\
\hline Tarea 40. Adivina los números & CD; SU & Tarea 46. Quito y pongo & CD; PO \\
Tarea 41. De diez en diez & CD; SU & Tarea 47. Completen tablas & CD; RC \\
Tarea 42. La tiendita de la escuela & CD & Tarea 48. Juguemos con tarjetas & CD \\
Tarea 43. ¿Cuánto dinero es? & EQ; PO & Tarea 49. ¿Cuánto le quito al 10? & CD \\
Tarea 44. Juguemos al cajero & EQ; PO; CD & Tarea 50. ¿Quién se acercó más? & - \\
Tarea 45. Encuentra la suma & CD & Tarea 51. ¿Con qué se midió? & - \\
\hline
\end{tabular}

Fueron identificadas representaciones de tipo numérico, icónico y tabular, y prevalecen las situaciones que evocan cantidades indeterminadas que se corresponden con los resultados de las operaciones del tipo $a+b=\mathbf{\square}$. Este tratamiento del signo igual como resultado también está presente en la tarea 42, que promueve la identificación de la mitad y el doble de un número en un escenario de problemas verbales. La analiticidad no está presente en las tareas de este bloque. Las tareas 40 y 41 se enmarcan en el conteo de los primeros 100 número naturales, centrándose en la ubicación de determinados números en la secuencia. La tarea 45 está centrada en la descomposición numérica únicamente en decenas y unidades para obtener un resultado específico, por lo cual no es posible que emerja la noción de equivalencia. Finalmente, las tareas 48 y 49 refieren al cálculo mental de la suma y resta de números menores a 10 . Por lo anterior, las tareas no fueron referidas como promotoras de un pensamiento algebraico. Identificamos que las tareas 43,44 y 46 potencian un pensamiento proto-algebraico de carácter estructural, mientras que la tarea 47 uno de carácter funcional. La formulación de la tarea 47 (Figura 11) fue planteada por los autores para ser resuelta por niños de 6 años que están iniciándose en el aprendizaje de la numeración y las operaciones de sumar y restar números de dos dígitos. 


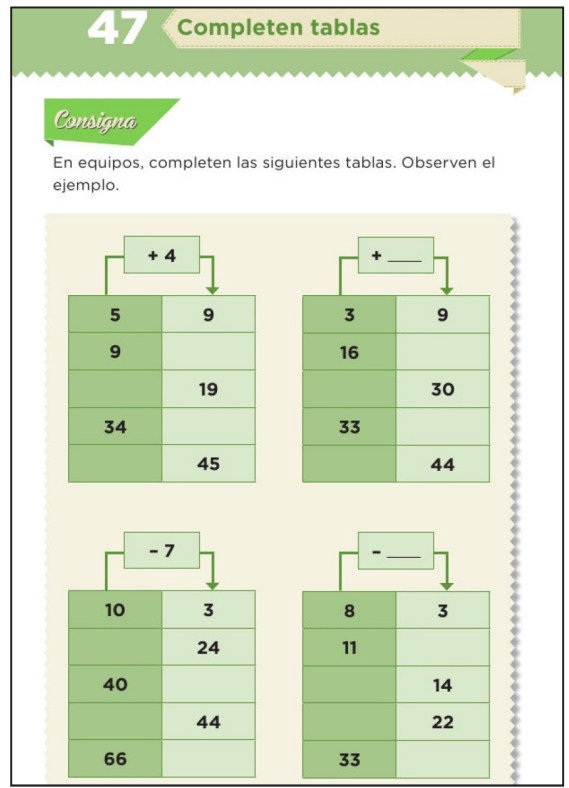

Figura 11. Ejemplo de tarea en el libro de texto de primaria (SEP, 2015: 88).

Aunque se advierte que la intención de la tarea es poner a los niños en situación de resolver una ecuación del tipo $x-7=24$, evitando el uso de símbolos literales para la cantidad desconocida, ya que esta es sustituida por una celda en blanco, él niño se ve orientado a interpretar el signo igual como resultado. Por tanto, solo se calcula el resultado aplicando la correspondiente consigna aditiva o sustractiva con números pequeños y particulares en cada una de las tablas de la tarea (sumar 4, sumar 6, restar 7 y restar 5). Con este planteamiento no se puede decir que intervenga el pensamiento algebraico, sino puramente aritmético. No obstante, con esta misma situación podría ampliarse la tarea para focalizar la atención en objetos y procesos de naturaleza proto-algebraica, según indicamos a continuación.

En la tarea de la Figura 13 se identifican conceptos como el de valor desconocido, relación de cambio entre dos cantidades y la regla de correspondencia. Implícitamente está la noción de función; por ejemplo, la tabla 3 (mirando de izquierda a derecha y de arriba hacia abajo) puede desarrollarse en el siguiente arreglo tabular (Figura 12): 
Completa la siguiente tabla:

\begin{tabular}{|c|c|c|c|c|c|c|c|c|c|c|c|c|c|c|c|c|c|c|c|c|c|}
\hline$x$ & 7 & 8 & 9 & & 11 & 12 & 13 & 14 & & & & & 19 & 20 & 21 & 22 & & 24 & 25 & & 27 \\
\hline$f(x)$ & 0 & 1 & & 3 & 4 & 5 & & 7 & 8 & & 10 & & 12 & 13 & & 15 & 16 & 17 & 18 & 20 \\
\hline
\end{tabular}

Figura 12. Tratamiento proto-algebraico funcional de la tarea propuesta en la Figura 11.

El maestro podría, entonces, promover el tránsito de lo numérico a lo tabular y posteriormente -en niveles educativos más avanzados- de lo gráfico a lo simbólico-literal. Si el alumno solo es capaz de identificar el término siguiente, se encuentra en un nivel cero de algebrización. Sin embargo, si se lo lleva a pensar en la idea de "cualquier posición", puede llegar a reconocer la regla de correspondencia y generalizar que "restando 7" a cualquier valor proporcionado (dominio de la función) puede obtener el valor correspondiente (contradominio de la función), lo cual supone un primer nivel de algebrización. De esta manera, con la ayuda del profesor, podría tener un primer encuentro con el pensamiento proto-algebraico funcional, con un nivel de algebrización 1. Finalmente, en niveles más avanzados, el niño podría llegar a formular una expresión más formal como $f(n)=n-7$ y ampliar el estudio de los naturales hacia los enteros, lo que implica un nivel de algebrización 2. Un nivel 3 podría alcanzarse en contextos más complejos donde se demanda operar con las variables (Puig \& Rojano, 2004) para obtener formas canónicas de expresión. Se puede apreciar cómo la tarea promueve la relación entre cantidades, escenario que el maestro de primaria puede aprovechar para promover el análisis de la situación planteada, a fin de que los alumnos piensen algebraicamente. En este sentido, las tareas deben estimular el uso de los números y operaciones numéricas como objetos para el pensamiento algebraico; hacer hincapié en el hecho de que los números pueden ser usados algebraicamente cuando el tipo o medida del número elegido requiere que el estudiante piense sobre la estructura y la relación entre cantidades, y no simplemente en operaciones aritméticas con cantidades (Kaput \& Blanton, 2002).

\subsection{ANÁlisis de LAS tAREAS DEL BLOQUE 5 DEL LIBRO DEL ALUMNO}

El bloque 5 del libro cuenta con 6 tareas donde encontramos objetos algebraicos tales como la noción de equivalencia (EQ) y propiedades de las operaciones (PO) en contextos numéricos. 
Tabla 6. Objetos algebraicos identificados en el quinto bloque del libro del alumno.

\begin{tabular}{|c|c|c|c|}
\hline Tareas & $\mathrm{OA}$ & Tareas & $\mathrm{OA}$ \\
\hline Tarea 52. iAlto! & $\mathrm{EQ} ; \mathrm{PO}$ & Tarea 55. Las cuentas de Carmita & EQ; PO \\
\hline Tarea 53. De todas las formas & EQ; PO & Tarea 56. La cajita mágica & $C D$ \\
\hline Tarea 54. Los regalos de Carmita & $C D$ & $\begin{array}{l}\text { Tarea 57. Juguemos "iBasta!" con } \\
\text { números }\end{array}$ & - \\
\hline
\end{tabular}

Detectamos registros del tipo numérico e icónico, la analiticidad continúa ausente en el tratamiento de cantidades indeterminadas. En este bloque las tareas 52, 53 y 55 resultaron favorecedoras para el desarrollo de un pensamiento proto-algebraico de tipo estructural. Por ejemplo, la tarea 55, representada en la Figura 13, está planteada para que el niño compare expresiones equivalentes de un mismo valor numérico. La particularidad de esta tarea es que solicita comparar dos procedimientos diferentes que conducen a un mismo resultado, a través de preguntas específicas que conllevan el focalizar las equivalencias en las expresiones justificadas por la descomposición numérica de los cálculos de Carmita y Lupe, respectivamente, es decir, comparar $14+30=15-1+29+1$ y $10+34=15-5+29+5$.

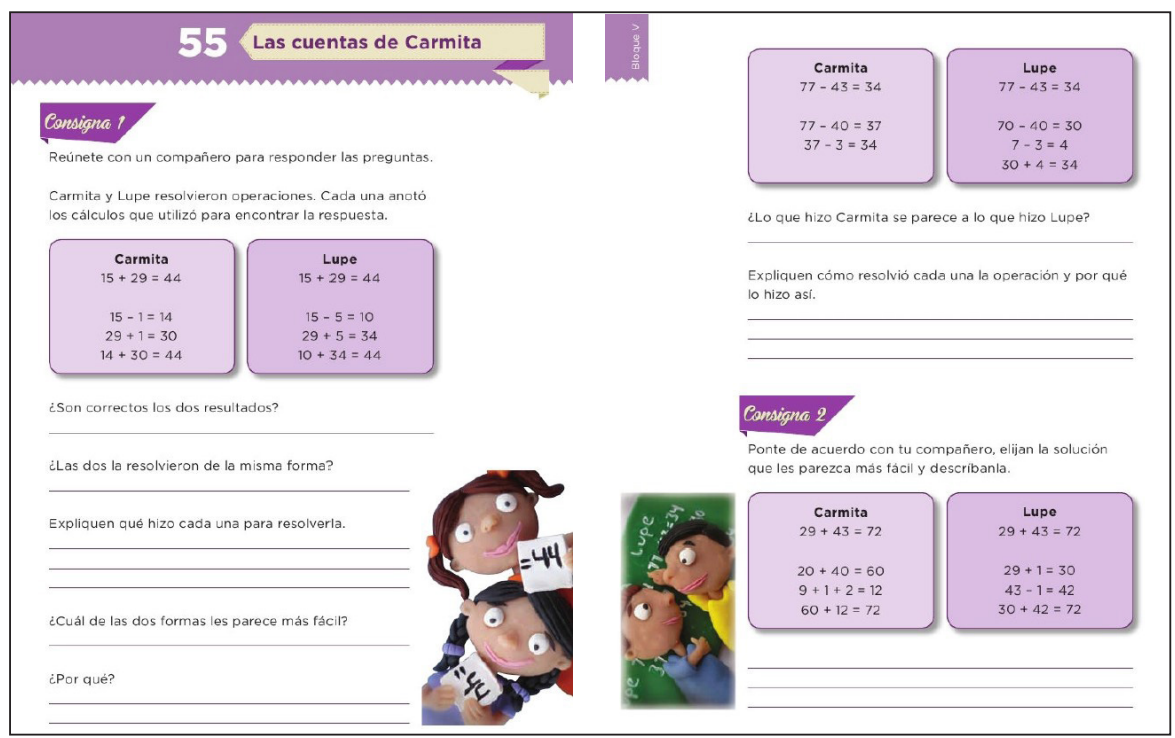

Figura 13. Ejemplo de tarea en el libro de texto de primaria (SEP, 2015: 97). 
Es posible incentivar, posteriormente, a reconocer la propiedad de que "dos cantidades iguales a una tercera son iguales entre si" al centrase en las expresiones $14+30=44$ y $10+34=44$ y, por tanto, $14+30=10+34$. Enfatizamos, otra vez, que el niño no precisa nombrar esta propiedad, sino más bien reconocerla a través de su uso. Este tipo de actividades se enmarcaría en un nivel 1 de algebrización, pues promueven un pensamiento proto-algebraico de carácter estructural.

Es importante señalar que en estos cinco bloques, como hemos reportado, identificamos tareas que presentan situaciones de secuencias numéricas, como las tareas 7, 9, 29, 30, 31, 32, 40 y 41. Sin embargo, las situaciones que plantean dichas tareas están encaminadas al conteo de al menos los primeros 100 números naturales, completando casillas vacías (cantidad desconocida) consecutivas. De manera similar, encontramos tareas en las que emergen únicamente cantidades indeterminadas, condición que resultó insuficiente para la reorientación de la tarea hacia niveles progresivos de algebrización.

De manera general, en la siguiente tabla se puede apreciar la identificación de los objetos algebraicos de índole estructural (OAE) o funcional (OAF), así como el tipo de notación o representación (NoR) que se utiliza en las tareas (T) por cada bloque (BL). Identificamos objetos proto-algebraicos estructurales como equivalencia (EQ), propiedades de las operaciones (PO) y cantidades desconocidas (CD); también otros de carácter funcional, por ejemplo, las relaciones de cambio (RC), patrones (PA) y sucesiones (SU). Todas las tareas tenían connotaciones verbales, pero también se identificaron aquellas representaciones icónicas (IC), numéricas (NU) y tabulares (TA) que eran necesarias para llevar a cabo la tarea. Es importante mencionar que la modelización (MO) solo estuvo presente a través de problemas de palabras que involucraban ecuaciones de la forma $a$ $+b=x$; en ninguna tarea se promueve operar con las cantidades desconocidas 0 indeterminadas. 
Tabla 7. Identificación de tareas favorecedoras de un pensamiento algebraico.

\begin{tabular}{|c|c|c|c|c|c|c|c|c|c|}
\hline \multirow{2}{*}{ - } & \multirow{2}{*}{$\frac{\text { Tarea }}{\mathrm{T} 7}$} & \multicolumn{3}{|c|}{$\mathrm{OAE}$} & \multirow{2}{*}{$\frac{\mathrm{OAF}}{\mathrm{SU}}$} & \multicolumn{2}{|c|}{ NoR } & \multirow[t]{2}{*}{ MO } & \multirow{2}{*}{$\begin{array}{c}\text { Pensamiento algebraico } \\
\text { que favorece }\end{array}$} \\
\hline & & & & $C D$ & & $\mathrm{NU}$ & TA & & \\
\hline & T9 & & & & SU & $\mathrm{NU}$ & TA & & - \\
\hline & $\mathrm{T} 10$ & & & & PA & IC & & & Funcional \\
\hline & $\mathrm{T} 12$ & EQ & & & & IC & & & Estructural \\
\hline & $\mathrm{T} 13$ & & & $C D$ & & & & MO & - \\
\hline & T14 & EQ & & & & IC & & & Estructural \\
\hline \multirow[t]{8}{*}{ BL2 } & T19 & EQ & $\mathrm{PO}$ & & & IC & & & Estructural \\
\hline & T20 & EQ & & & & IC & & & Estructural \\
\hline & T21 & EQ & PO & & & IC & & & Estructural \\
\hline & T22 & & & $C D$ & & & & MO & - \\
\hline & $\mathrm{T} 23$ & & & $C D$ & & & & & - \\
\hline & T24 & & & $C D$ & & & & MO & - \\
\hline & T26 & & & $C D$ & & & & & - \\
\hline & T28 & & & $C D$ & & IC & & MO & - \\
\hline \multirow[t]{10}{*}{ BL3 } & T29 & & & & $\mathrm{SU}$ & & $\mathrm{TA}$ & & - \\
\hline & T30 & & & $C D$ & SU & & TA & & - \\
\hline & T31 & & & & SU & & & & - \\
\hline & T32 & & & $C D$ & SU & $\mathrm{NU}$ & TA & & - \\
\hline & T33 & EQ & PO & $C D$ & & $\mathrm{NU}$ & $\mathrm{TA}$ & & Estructural \\
\hline & T34 & EQ & PO & $C D$ & & & & & Estructural \\
\hline & T35 & & & $C D$ & & & & MO & - \\
\hline & T36 & & & $C D$ & & & & MO & - \\
\hline & T37 & & & $C D$ & & & & MO & - \\
\hline & T38 & & $\mathrm{PO}$ & & & & & & Estructural \\
\hline \multirow[t]{2}{*}{ BL4 } & T40 & & & $C D$ & SU & $\mathrm{NU}$ & TA & & - \\
\hline & T41 & & & $C D$ & SU & & & & - \\
\hline
\end{tabular}




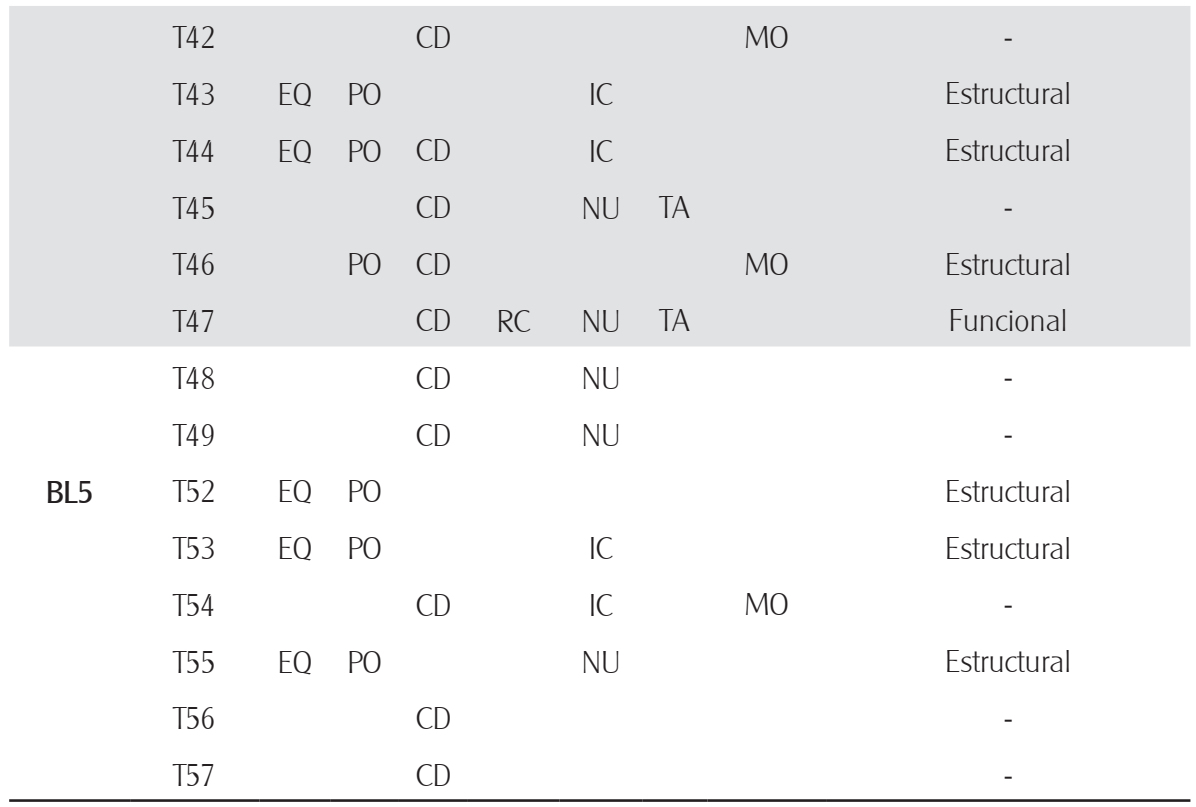

Lo expuesto, en el análisis de las tareas presentes en el libro de texto de primero de primaria, evidencia que la potencialidad de la tarea para promover el pensamiento algebraico en los estudiantes depende de la práctica matemática que pueda elaborarse a partir de ella. Se trata de posibles enfoques para las tareas propuestas en la educación primaria, con el propósito de hacer emerger elementos algebraicos. El análisis efectuado resulta útil en la formación inicial y continua del profesorado de esos grados educativos, ya que según la propuesta "Early algebra" y la "algebrización del currículo", los docentes han de suscitar la observación de patrones y propiedades matemáticas para crear un ambiente escolar en el cual se promueva que los alumnos exploren, discutan, argumenten y comprueben ideas (Kaput, 1998; Cai \& Knuth, 2011). Esto implica el desarrollo de competencias específicas que debieran ser consideradas en la formación de los maestros, si la intención es el desarrollo del razonamiento algebraico en los niños. Así, la propuesta de niveles de algebrización para el análisis de tareas podría incorporarse a ciclos formativos adecuados para los docentes, que les permitan desarrollar orientaciones instruccionales para el aula, con la finalidad de favorecer el surgimiento y consolidación de niveles progresivos del razonamiento algebraico en los niños. 


\section{REFLEXIONES FINALES}

El conflicto que representa el aprendizaje del álgebra para los estudiantes implica el desafío de encontrar nuevas maneras de hacer que esta materia esté a disposición de todos los escolares. Para tal fin, las tareas implementadas en el aula juegan un papel crucial si se quiere algebrizar el currículo de primaria (Kaput, 1998), pues cuando el libro de texto es un documento básico en la planificación de clase del profesor, es importante conocer la pertinencia de su contenido, y en qué grado las tareas en él propuestas contribuyen al desarrollo de la competencia matemática, en este caso el desarrollo del pensamiento algebraico en los alumnos.

El análisis del libro del alumno de primero de primaria refleja que las tareas no están intencionalmente dirigidas a promover un pensamiento algebraico en los niños, y que se prioriza el registro numérico. Sin embargo, a través de los niveles de algebrización identificamos los objetos algebraicos implícitos en las tareas que las categorizaron como de tipo estructural o funcional, lo cual permite una orientación hacia formas de pensamiento proto-algebraico; es claro que no todas las tareas están en posibilidad de una reorientación o modificación para tal fin. De este modo detectamos que aquellas tareas de las que emerge la noción de equivalencia, la idea de patrón como unidad de repetición a partir de la cual se puede generalizar, y aquellas en las cuales se establece un análisis de relaciones de cambio entre cantidades, son potencialmente promotoras de un pensamiento proto-algebraico. Los diferentes registros o representaciones favorecen el análisis para una generalización, y marcan la pauta para diferenciar niveles proto-algebraicos en la actividad matemática. El tratamiento o cálculo analítico diferencia entre un desarrollo del pensamiento proto-algebraico del algebraico que, como se evidenció, también tiene lugar en las tareas de este nivel educativo, si se enfoca de manera adecuada. Asimismo, la modelización de problemas verbales queda asociada a expresiones de la forma $A+B_{1}=x$ promoviendo el significado del signo igual como resultado. Del estudio del libro de texto emergió que la cantidad indeterminada o desconocida como objeto algebraico no es indicativo suficiente para orientar al niño hacia un pensamiento proto-algebraico; del mismo modo, las secuencias numéricas o de figuras en las que no se incentiva a la generalización, tampoco facilitan dicha orientación. Lo anterior detona la idea de que existen objetos algebraicos fundamentales cuya presencia en las tareas potencia el desarrollo de un pensamiento algebraico. Resulta evidente que no es posible desvincular la tarea de la práctica matemática 
que implica, porque a partir de esta se establece su potencialidad para promover un pensamiento algebraico.

La distinción de tareas que promueven o no un razonamiento algebraico puede ser útil en la formación matemática de maestros de educación primaria y secundaria, dado que deben ser capaces de valorar la complejidad de las tareas planteadas en los libros de texto, analizarlos, compararlos y elegir adecuadamente aquellas que podrían ser utilizadas para explotar las ideas matemáticas reconocidas como "algebraicas" y examinar las características de estas tareas, aunque no parezcan algebraicas a simple vista. Este hecho cobra mayor relevancia porque si se considera que diversas investigaciones informan que los niños ciertamente pueden resolver tareas tradicionalmente consideradas propias del álgebra (Carpenter, Frankle \& Levi, 2003; Carraher, Schliemann, Brizuela \& Earnest, 2006; Radford 2010; Castro, Cañadas \& Molina, 2017), entonces se requiere que los profesores de todos los niveles de educación primaria sean capaces de promover el pensamiento algebraico a través de las tareas que plantean en el aula. Sin embargo, fomentar un aprendizaje con comprensión, introduciendo el carácter algebraico en la matemática elemental, requiere llevar a cabo procesos formativos con los docentes (Carraher \& Schliemann, 2007; Branco \& Ponte, 2012).

La herramienta desarrollada a partir del Enfoque Onto-Semiótico no solo proporciona elementos que permiten entender la aproximación del álgebra escolar a los niveles elementales, sino que también facilita determinar el carácter algebraico de las tareas, posibilitando que el maestro de primaria seleccione y modifique aquellas que proporcionen un mayor grado de algebrización de la actividad matemática de los alumnos.

\section{REFERENCIAS}

Aké, L. P., Godino, J. D., Gonzato, M. y Wilhelmi, M. R. (2013). Proto-algebraic Levels of Mathematical Thinking. En: A. Lindmeier \& A. Heinze (eds.), Proceedings of the $37^{\text {th }}$ Conference of the International Group for the Psychology of Mathematics Education -PME, Vol. 2, (pp. 1-8). Kiel, Germany: Leibniz-Institut.

Alibali, M. W., Knuth, E. J., Hattikudur, S., McNeil, N. M. \& Stephens, A. C. (2007). A Longitudinal Examination of Middle School Students' Understanding of the Equal Sign and Equivalence Equations. Mathematical Thinking and Learning, 9 (3), pp. 221-247. 
Castro, E., Cañadas, M. C. \& Molina, M. (2017). Pensamiento funcional mostrado por estudiantes de Educación Infantil. Edma 0-6: Educación Matemática en la Infancia, 6(2), 1-13.

Blanton, M. L. \& Kaput, J. J. (2011). Functional Thinking as a Route into Algebra in the Elementary Grades. En: J. Cai \& E. Knuth (eds.). Early Algebraization: A Global Dialogue from Multiple Perspectives (pp. 5-23). Berlin, Germany: Springer.

Branco, N. y Ponte, J. P. (2012). Developing Algebraic and Didactical Knowlendge in Pre-service Primary Teacher Education. En T. Y. Tso (ed.). Proceedings of the $36^{\text {th }}$ Conference of the International Group for the Psychology of Mathematics Education, - PME, Vol. 2 (pp. 75-82). Taipei, Taiwan: PME.

Butto, C. \& Rojano, T. (2004). Introducción temprana al pensamiento algebraico: Abordaje basado en la geometría. Educación Matemática, 16 (1), pp. 113-148.

Cai, J. \& Knuth, E. (2011). Early Algebraization: A Global Dialogue from Multiple Perspectives. Heidelberg, Germany: Springer-Verlag.

Carpenter, T. P., Franke, M. L. \& Levi, L. (2003). Thinking Mathematically. Integrating Arithmetic and Algebra in Elementary School. Portsmouth, New Hampshire: Heinemann.

Carraher, D. W. \& Schliemann, A. L. (2007). Early Algebra and Algebraic Reasoning. En F. Lester (ed.). Second Handbook of Research on Mathematics Teaching and Learning, Vol. 2 (pp. 669-705). Charlotte, North Carolina: Information Age Publishing, Inc. \& NCTM. Carraher, D., Schliemann, A. D. \& Brizuela, B. M. (2007). Early Algebra, Early Arithmetic: Treating Operations as Functions. En: M. L. Fernández (ed). Conferencia magistral presentada en el $22^{\text {nd }}$ Annual Meeting of the North American Chapter of the International Group for the Psychology of Mathematics Education - PME-NA, Tucson, Arizona.

Carraher, D. W., Schliemann, A. D., Brizuela, B. M. \& Earnest, D. (2006). Arithmetic and Algebra in Early Mathematics Education. Journal for Research in Mathematics Education, 37(2), 87-115.

Carraher, D. W., Schliemann, A. D. \& Schwartz, J. L. (2008). Early Algebra Is not the Same as Algebra Early. En: J. Kaput. D. Carraher y M. Blanton (eds.). Algebra in the Early Grades (pp. 235-272). Mahwah, New Jersey: Erlbaum.

Ellis, A. (2011). Algebra in the Middle School: Developing Functional Relationships Through Quantitative Reasoning. En: T. Rowland y K. Ruthven. (eds.). Mathematical Knowledge in Teaching (pp. 9-25). London: Springer.

Filloy, E., Puig, L. y Rojano, T (2008). Educational Algebra. A Theorical and Empirical Approach. New York: Springer.

Godino, J. D., Aké, L. P., Gonzato, M. \& Wilhelmi, M. R. (2014). Niveles de algebrización de la actividad matemática escolar. Implicaciones para la formación de maestros. Enseñanza de las Ciencias, 32 (1), pp. 199-219. 
Godino, J.D., Neto, T. Wilhelmi, M.R., Aké, L., Etchegaray, S. \& Lasa, A. (2015). Niveles de algebrización de las prácticas matemáticas escolares. Articulación de las perspectivas ontosemiótica y antropológica. Avances de Investigación en Educación Matemática, 8, 117-142.

Godino, J. D., Batanero, C. \& Font, V. (2007). The Onto-Semiotic Approach to Research in Mathematics Education. Zentralblatt für Didaktik der Mathematik (ZDM): The International Journal on Mathematics Education, 39 (1), pp. 127-135.

Godino, J. D., Castro, W. F., Aké, L. P. \& Wilhelmi, M. R. (2012). Naturaleza del razonamiento algebraico elemental. Boletim de Educação Matemática - BOLEMA, 26 (42B), 483-511.

Hoch, M. \& Dreyfus, T. (2004). Structure Sense in High School Algebra. The Effect of Brackets. En: M. J. Hoines \& A. B. Fuglestad (eds). Proceedings of the $28^{\text {th }}$ Conference of the International Group for the Psychology of Mathematics Education - PME, Vol. 3, (pp. 49-56). Bergen, Norway: Bergen University College.

Jamieson-Proctor, R. \& Byrne, C. (2008). Primary Teachers' Beliefs about the Use of Mathematics Textbooks. En: M. Goos, R. Brown \& K. Makar (eds.). Proceedings of the $31^{\text {st }}$ Annual Conference of the Mathematics Education: Meeting Research Group of Australasia-MERGA31: Navigating Currents and Charting Directions (pp. 295-302). Brisbane, Australia: MERGA.

Jones, I. \& Pratt, D. (2006). Three Utilities for the Equal Sign. En: H. L. Chick \& J. L. Vincent (eds.). Proceedings of the $29^{\text {th }}$ International Group for the Psychology of Mathematics Education - PME, Vol. 3 (pp.185-192). Melbourne, Australia: University of Melbourne.

Kaput, J. (1998). Teaching and Learning a New Algebra. En: E. Fennema y T. A. Romberg (eds.). Mathematics Classrooms that Promote Understanding (pp. 133-155). Mahwah, New Jersey: Lawrence Erlbaum Associates.

Kaput, J. \& Blanton, M. L. (2002). Design Principles for Tasks that Support Algebraic Thinking in Elementary School Classrooms. En: A. D. Cockburn y E. Nardi (eds.). Proceedings of the $26^{\text {th }}$ Annual Conference of the International Group for the Psychology of Mathematics Education - PME, Vol. 2 (pp. 105-112). Norwich, England: University of East Anglia.

Kieran, K., Pang, J., Schifter, D. \& Fong, S. (2016). Early Algebra. Research into its Nature, its Learning, its Teaching. Hamburg, Germany: Springer.

Lins, R. \& Kaput, J. J. (2004). The Early Development of Algebraic Reasoning: The Current State of the Field. En K. Stacey, H. Chick y M. Kendal (eds.). The Future of the Teaching and Learning of Algebra. The $12^{\text {th }}$ ICMI Study (pp. 47-70). Massachusetts, USA: Kluwer Academic Publishers. 
National Council of Teachers of Mathematics (2000). Principles and Standards for School Mathematics. Reston, VA: NCTM

National Governors Association Center for Best Practices and the Council of Chief State School Officers. (2011). Common Core State Standards for Mathematics-CCSSM. Disponible en http://www.corestandards.org/assets/CCSSI_Math\%20Standards.pdf. Puig, L. \& Rojano, T. (2004). The History of Algebra in Mathematics Education. En: K. Stacey, H. Chick y M. Kendal (eds.). Proceedings of the $12^{\text {th }}$ International Commission on Mathematical Instruction-ICMI Study Conference: The Future of the Teaching and Learning of Algebra (pp. 189- 224). London: Kluwer Academic Publishers.

Radford, L. (2003). Gestures, Speech, and the Sprouting of Signs: A Semiotic-cultural Approach to Students' Types of Generalization. Mathematical Thinking and Learning, 5(1), pp. 37-70.

Radford, L. (2010). The Eye as a Theoretician: Seeing Structures in Generalizing Activities. For the Learning of Mathematics, 30(2), 2-7.

Radford, L. (2012). Early Algebraic Thinking: Epistemological, Semiotic, and Developmental Issues. En S. J. Cho (ed.). Proceedings of the $12^{\text {th }}$ International Congress on Mathematical Education- ICME (pp. 675-694). Seúl, Korea: National University of Education.

Rivera, (2013). Types and Levels of Pattern Generalization. Teaching and Learning Patterns in School Mathematics. Psychological and Pedagogical Considerations, 55-92. Springer: New York.

Schliemann, A. D., Carraher, D. W. \& Brizuela, B. (2007). Bringing Out the Algebraic Character of Arithmetic: From Children's Ideas to Classroom Practice. Mahwah, New Jersey: Lawrence Erlbaum Associates.

Secretaría de Educación Pública (2011). Plan de estudios 2011. Educación básica. México: SEP. Disponible en http://issuu.com/dgeb/docs/planedu2011?e=3503076/2622744.

Secretaría de Educación Pública (2015). Desafíos matemáticos 2015. Libro para el alumno. Educación Básica Primaria. Primer grado. México: SEP.

Vasilachis, I. (2009). Estrategias de investigación cualitativa. España: Gedisa.

Warren, E. (2001). Algebraic Understanding and the Importance of Operation Sense. En: M. Heuvel-Penhuizen (ed.). Proceedings of the $25^{\text {th }}$ International Group for the Psychology of Mathematics Education - PME, Vol. 4 (pp. 399-406). Utrecht, The Netherlands: Freudenthal Institute.

Whitacre, I., Schoen, R. C., Champagne, Z. \& Goddard, A. (2017). Relational Thinking: What's the Difference? Teaching Children Mathematics, 23 (5), pp. 302-308. 